\title{
Factors Associated with Health-Related Quality of Life in Women with Breast Cancer in the Middle East: A Systematic Review
}

\author{
Rana El Haidari 1,2,*, Linda Abou Abbas ${ }^{3}{ }^{(0)}$, Virginie Nerich ${ }^{2,4}$ and Amélie Anota 2,5,6 \\ 1 Environments and Health doctoral school, University of Bourgogne Franche-Comté, 25000 Besançon, France \\ 2 INSERM (French Institut of Health and Medical Research), EFS BFC (Etablissement français du sang \\ Bourgogne franhce-comté), UMR1098 (Interactions Greffon-Hôte-Tumeur/Ingénierie Cellulaire et Génique), \\ University of Bourgogne Franche-Comté, RIGHT Interactions Greffon-Hôte-Tumeur/Ingénierie Cellulaire et \\ Génique, F-25000 Besançon, France; v1nerich@chu-besancon.fr (V.N.); aanota@chu-besancon.fr (A.A.) \\ 3 Neuroscience research center, Faculty of medical sciences, Lebanese university, 1001 Beirut, Lebanon; \\ l.abouabbas@ul.edu.lb \\ 4 Department of Pharmacy, University Hospital of Besançon, 25030 Besançon, France \\ 5 Methodology and Quality of Life in Oncology Unit, University Hospital of Besançon, 25000 Besançon, France \\ 6 French National Platform Quality of Life and Cancer, 250000 Besançon, France \\ * Correspondence: rana.el_haidari@univ-fcomte.fr or ranahaidari14@hotmail.com; Tel.: +96-1-7166-2058
}

Received: 3 February 2020; Accepted: 9 March 2020; Published: 16 March 2020

\begin{abstract}
Objectives: The aim of the present systematic review was to identify the factors that potentially influence health-related quality of life (HRQoL) in women with breast cancer (BC) in the Middle East. Methods: A systematic search of the PubMed, Ovid Medline, Cochrane, Embase, Cumulative Index to Nursing and Allied Health Literature (CINAHL), Scopus, and Ebscohost databases was conducted to identify all relevant articles published in peer-reviewed journals up to April 2018. The keywords were "Health related quality of life", "Breast Cancer", and "Middle East countries". The Newcastle-Ottawa (NOS) scale was used to evaluate the methodological quality of the included studies. Due to the methodological heterogeneity of the identified studies, no statistical pooling of the individual effect estimates was carried out; instead, the results were summarized descriptively. Results: A total of 5668 articles were screened and 33 studies were retained. The vast majority of these studies were cross-sectional and only two were longitudinal prospective studies. Concerning the methodological quality, only 39\% were of high quality. Our comprehensive literature review identified several modifiable and non-modifiable risk factors associated with HRQoL, including sociodemographic, clinical, and treatment-related factors as well as behavioral and psychosocial factors. Conclusion: This study has many implications for clinical practice and may provide a framework for establishing policy interventions to improve HRQoL among women with BC. Healthcare systems in the Middle East are encouraged to develop interventional programs targeting modifiable factors, particularly socio-demographic, behavioral, and psychosocial factors.
\end{abstract}

Keywords: Breast Cancer; Health-related quality of life; Systematic review; Middle East; Women

\section{Introduction}

Globally, breast cancer (BC) is the most commonly occurring cancer in women [1]. The worldwide GLOBOCAN estimates revealed that 2,088,849 new BC cases and 626,679 cancer-related deaths occurred in 2018, with a projected number of over 3,059,829 women to be diagnosed in 2040 [1]. This reflects a significant global increase of $46 \%$, albeit with significant differences in incidence between countries [1]. 
The Middle East is a region located in western Asia and extends into North Africa, with an estimated population of over 411 million. It comprises 15 countries with different ethnic groups holding different cultures, norms, and beliefs. Although BC incidence rates are lower in the Middle East compared to other western societies, they have substantially increased over the last few decades [1]. According to GLOBOCAN estimates, approximately 119,985 (34.2\%) new BC cases and 48,661 (24.9\%) cancer-related deaths occurred in 2018 [1]. Furthermore, a large proportion of women are diagnosed with $B C$ at younger age, i.e., under 40 years old, and at an advanced stage, where BC is generally aggressive and requires mastectomy [2]. In most countries of the Middle East, BC is still considered a sensitive and taboo topic, surrounded by shame and silence. Most Middle Eastern societies mention it as "that other disease" and many women are petrified to talk about it [3]. The perception of BC as incurable and its fatality are also a concern. Therefore, a diagnosis of $\mathrm{BC}$ can be a devastating event and can expose Middle Eastern women to a high burden of psychological suffering that could adversely affect their health-related quality of life (HRQoL).

According to Osoba et al., HRQoL is "a multidimensional construct that includes perceptions of both positive and negative aspects of dimensions such as physical, emotional, social and cognitive functions. It also includes the negative aspects of somatic discomfort and other symptoms produced by a disease or its treatment" [4]. HRQoL is considered one of the main determinants of treatment success in modern oncology $[5,6]$. Findings from research suggest that assessment of HRQoL as part of clinical practice has the potential to improve the quality of care that patients receive, as well as their health status. Thus, its evaluation has become as important as survival in making treatment decisions [7].

Over the last few decades, evaluation of HRQoL and its associated factors in patients diagnosed with $\mathrm{BC}$ have witnessed increasing interest among both researchers and decision-makers [8]. Studies from western societies have identified several factors affecting HRQoL in women with BC. Those factors include socio-demographic characteristics, such as age [9], marital status, economic problems [10], and the experience of daily difficulties in work [11], as well as clinical and psychological characteristics of BC survivors, such as stage of disease [12], pain, stress [13], depression and anxiety [14], social relationships [15], variations in self-confidence, self-efficacy [16], and the development of harmful sentiments. In addition, HRQoL was found to be affected by the therapeutic procedures used [17].

A recent review and meta-analysis evaluating the QoL of patients with BC in the Middle East revealed that fewer than one-third of patients (21\%) had good QoL [18]. However, studies on factors associated with HRQoL in patients with BC in Middle Eastern countries are scarce. Findings from western societies cannot be extrapolated to BC patients in the Middle East countries, which have a distinct lifestyle and culture. Several potential issues may contribute to the expected disparity, including socioeconomic difficulties, limited access to health care, lack of supportive care, late BC diagnosis, self-perception of the disease, social constraints, and other religious/cultural restrictions [3]. Identification of the factors associated with HRQoL could help professionals to develop effective health interventions and specific approaches to promote quality of life in BC patients in the Middle East. Therefore, there is a need for a synthesis of existing research to aid in the design of interventions to improve HRQoL. In this context, the aim of the present study is to perform a systematic review to identify the factors associated with HRQoL in the countries of the Middle East.

\section{Materials and Methods}

The systematic review was performed in accordance with the Preferred Reporting Items for Systematic reviews and Meta-Analyses guidelines (PRISMA) published in 2009 [19]. An ethics statement was not required.

\subsection{Data Sources and Search Strategy}

A systematic search of the following databases was conducted: PubMed, Ovid Medline, Cochrane, Embase, Cumulative Index to Nursing and Allied Health Literature (CINAHL), and Ebscohost, to identify all relevant articles published in peer-reviewed journals up to April 2018. Keywords related to 
"Health related quality of life", "Breast Cancer", and "Middle East countries" were combined using Boolean operators ("AND", and "OR"). The search combination of keywords and Medical Subject Headings (MeSH) terms is presented in Table 1. Bibliography lists from all eligible articles were also hand searched to identify additional papers potentially relevant for inclusion.

Table 1. Medical Subject Headings (MeSH) terms used in the search strategy.

\begin{tabular}{ccc}
\hline MeSH Terms for HRQoL & MeSH Terms for Breast Cancer & MeSH Terms for ME Countries \\
\hline & Breast Neoplasms OR Breast & Middle East OR Bahrain OR Egypt \\
quality of life OR health related & Neoplasm OR Breast Cancer OR & OR Iran OR Iraq OR Jordan OR \\
quality of life OR life style OR & Breast Carcinoma OR Breast OR & Kuwait OR Lebanon OR Oman \\
well-being OR health status & mammary carcinoma OR cancer & OR Palestine OR Qatar OR Saudi \\
& OR mammary cancer OR & Arabia OR Syria OR Turkey OR \\
& carcinoma mammae & United Arab Emirates OR Yemen \\
\hline
\end{tabular}

\subsection{Eligibility Criteria}

Articles were included if they met all of the following criteria:

1. Population: women diagnosed with BC only;

2. Setting: studies conducted in one or more Middle East countries;

3. Outcome: HRQoL assessed using any valid and reliable instrument that measures quality of life, such as EORTC QLQ-C30: European Organization for Research and treatment of Cancer-Quality of Life Questionnaire; BR23: Breast cancer specific module; FACT-B: The Functional Assessment of Cancer Therapy—Breast Cancer; FACT-G: The Functional Assessment of Cancer Therapy-General Questionnaire; NMCBRI-Q: the national medical center and Beckman research institute questionnaire; LQI: Life quality inventory; QOL-BC: The American quality of life BC; QoLS: The Quality of Life Scale; SF-36: Short-Form Health Survey Questionnaire; WHOQOL-BREF: WHO questionnaire of quality of life;

4. Experimental or observational study investigating any associated factors with HRQoL;

5. English language articles.

Articles were excluded if they met any one or more of the following criteria:

1. Narrative or systematic reviews;

2. Editorials, expert opinions, comments (commentary), methodological article, or conference abstracts and proceedings;

3. Assessment of the effect of specific interventions on HRQoL, such as sport, exercise, yoga, or focus groups.

\subsection{Data Extraction and Synthesis}

Titles and abstracts identified from searches were screened for relevance, and duplicates were excluded. The full texts of all relevant articles were retrieved and their eligibility for inclusion was assessed.

Two reviewers (R.H. and L.A.A.) performed data extraction independently. Any disagreements were resolved by discussion and by cross-checking the papers. The following information was recorded from each paper: basic study information (last author's name, publication year, and country), study design, sample characteristics (sample size and age), clinical data (BC stage and treatment), HRQoL questionnaire used and time of assessment, as well as the results of the studies, including global HRQoL (Mean and SD), determinants, and signs of the association.

\subsection{Methodological Quality Assessment}

The methodological quality and risk of bias of the included studies was assessed independently by two reviewers (R.H. and L.A.A.) using the Newcastle-Ottawa scale (NOS), a quality assessment 
tool [20]. Ratings are made using a "star" system to assess the quality of a study for eight items grouped into three domains: (1) selection of participants, (2) comparability of study groups, and (3) verification of the exposure or the outcome of interest. According to the NOS scale, a maximum of nine stars can be allocated for case-control or longitudinal studies and a maximum of six stars can be obtained for cross-sectional studies. Case-control or longitudinal studies that score five or more stars and cross-sectional studies with four or more stars are considered to be of high quality.

\section{Results}

\subsection{Study Selection}

The literature search identified a total of 5668 records. After the exclusion of duplicate records and articles with non-relevant title and abstracts, 5227 published studies were retained for screening. Screening of titles and abstracts identified 137 potentially eligible articles. Full-text analyses of these 137 articles identified 56 relevant articles, of which 33 finally met the eligibility criteria for inclusion in the present review (Figure 1).

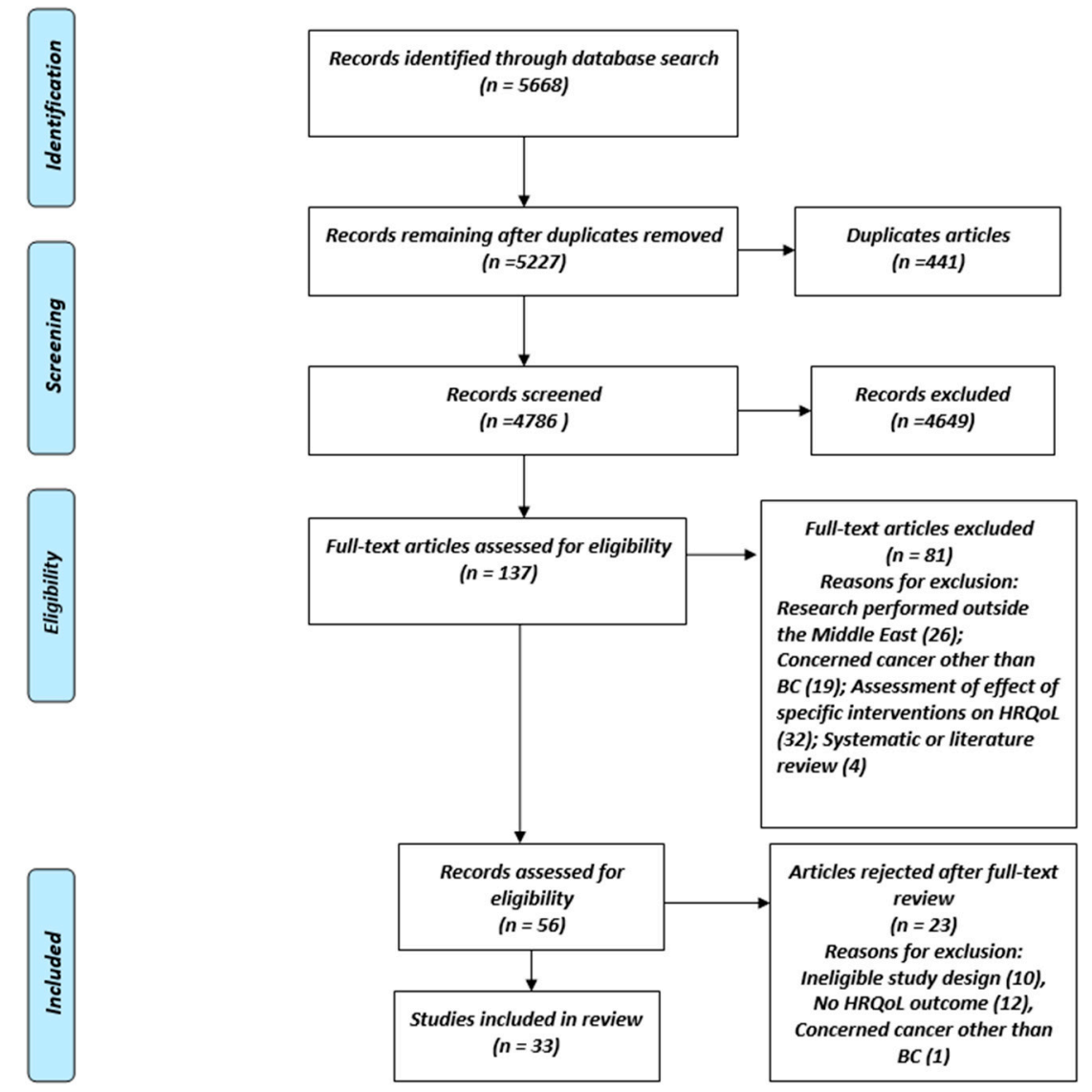

Figure 1. Flow chart of study selection strategy.

\subsection{Characteristics of the Included Studies}

The characteristics of the articles included in the present systematic review are summarized in Table 2. Almost half of the studies were conducted in Iran $(n=15,45 \%)$, followed by Turkey $(n=9$, $27 \%)$, Saudi Arabia $(n=3,9 \%)$, Jordan $(n=2,6 \%)$, and one each (3\%) from Lebanon, Kuwait, Bahrain, and Yemen (Table 2). More than half of the studies in were from Cancer Centers $(n=17,52 \%)$ and the rest $(n=16,48 \%$ ) were conducted in hospitals. First authors of the published studies were mainly affiliated with universities. Articles were published in medical (42\%) or medical oncology journals $(18 \%)$. About two-thirds of the studies (64\%) used interviews as a method to collect data. Concerning 
study designs, the majority had a cross-sectional design $(n=28,85 \%)$. Three studies used a case-control design, while only two were longitudinal studies.

Table 2. Characteristics of the 33 studies included in the systematic review.

\begin{tabular}{|c|c|c|}
\hline Characteristic & Number & Percentage \\
\hline \multicolumn{3}{|c|}{ Country } \\
\hline Iran & 15 & 45 \\
\hline Turkey & 9 & 27 \\
\hline Saudi Arabia & 3 & 9 \\
\hline Jordan & 2 & 6 \\
\hline Lebanon & 1 & 3 \\
\hline Kuwait & 1 & 3 \\
\hline Bahrain & 1 & 3 \\
\hline Yemen & 1 & 3 \\
\hline \multicolumn{3}{|c|}{ Location of study } \\
\hline Cancer center & 17 & 52 \\
\hline Hospital & 16 & 48 \\
\hline \multicolumn{3}{|c|}{ Main location of first author } \\
\hline University & 27 & 82 \\
\hline Hospital & 3 & 9 \\
\hline Research center & 2 & 6 \\
\hline Medical center & 1 & 3 \\
\hline \multicolumn{3}{|c|}{ Type of journal } \\
\hline Medical & 14 & 42 \\
\hline Medical oncology & 6 & 18 \\
\hline Multidisciplinary & 4 & 12 \\
\hline Clinical & 4 & 12 \\
\hline Academic & 4 & 12 \\
\hline Clinical psychology & 1 & 3 \\
\hline \multicolumn{3}{|c|}{ Type of data collection } \\
\hline Self-reported & 12 & 36 \\
\hline Interview & 21 & 64 \\
\hline \multicolumn{3}{|c|}{ Study design } \\
\hline Cross-sectional & 28 & 85 \\
\hline Case-control & 3 & 9 \\
\hline Longitudinal & 2 & 6 \\
\hline
\end{tabular}

\subsection{Description of the Selected Atudies}

A detailed description of the included studies is presented in Table 3. The majority of the women participating in the studies had stage II BC and were treated with chemotherapy. 
Table 3. Description of the selected studies.

\begin{tabular}{|c|c|c|c|c|c|c|c|}
\hline \multirow{2}{*}{$\begin{array}{l}\text { Author Name, } \\
\text { Country-Year of } \\
\text { Publication }\end{array}$} & \multirow[t]{2}{*}{ Type of Study } & \multirow[t]{2}{*}{ Sample Size } & $\begin{array}{l}\text { Age of BC } \\
\text { Patients }\end{array}$ & Stage & Treatment & \multirow[t]{2}{*}{ Time of Assessment } & \multirow[t]{2}{*}{ Questionnaires } \\
\hline & & & Mean (SD) & $\%$ & $\%$ & & \\
\hline $\begin{array}{l}\text { Ahmad A [21], } \\
\text { Saudi } \\
\text { Arabia-2017 }\end{array}$ & Cross-sectional & 145 & $50.3(13.5)$ & $\begin{array}{l}\text { I and II: } 61.2 \\
\text { III and IV: } 38.8\end{array}$ & $\begin{array}{l}\text { Cancer surgery: } 64.8 \\
\text { Chemotherapy: } 73.1 \\
\text { Radiotherapy: } 57.9 \\
\text { Immunotherapy: } 49\end{array}$ & $\begin{array}{c}\text { First year after cancer \% } \\
\text { Yes: } 52.4 \\
\text { No: } 47.6\end{array}$ & SF-36 \\
\hline $\begin{array}{c}\text { Ahrafizadeh H } \\
\text { [22], Iran-2017 }\end{array}$ & Cross-sectional & 100 & $46.8(11.5)$ & - & $\begin{array}{c}\text { Mastectomy: } 58 \\
\text { Breast conservative surgery: } 31 \\
\text { Chemotherapy: } 100\end{array}$ & During chemotherapy & WHOQOL-BREF \\
\hline $\begin{array}{c}\text { Akel R [23], } \\
\text { Lebanon-2017 }\end{array}$ & Cross-sectional & $\begin{array}{l}150 \text { (121 Lebanese, } \\
26 \text { Iraqi, } 6 \text { other) }\end{array}$ & $53.5(10.4)$ & $\begin{array}{l}\text { I: } 29.3 \\
\text { II: } 38.7 \\
\text { III: } 23.3 \\
\text { IV: } 8.7\end{array}$ & $\begin{array}{l}\text { Chemotherapy: } 79.3 \\
\text { Radiotherapy: } 80.7 \\
\text { Surgery: } 97.3 \\
\text { Hormone therapy: } 86\end{array}$ & $\begin{array}{c}0.5 \text { to } \leq 2 \text { years: } 13.3 \\
3 \text { to } \leq 5 \text { years: } 68.7 \\
\geq 5 \text { years: } 18\end{array}$ & FACT-B \\
\hline $\begin{array}{l}\text { Akin S [24], } \\
\text { Turkey-2008 }\end{array}$ & Longitudinal & 141 & $46.9(10.1)$ & $\begin{array}{c}\text { I: } 6.4 \\
\text { II: } 47.5 \\
\text { III: } 31.2 \\
\text { IV: } 14.29 \\
\end{array}$ & $\begin{array}{l}\text { Chemotherapy: } 100 \\
\text { Surgery: } 76.6\end{array}$ & $\begin{array}{l}\text { Pre and Post } \\
\text { chemotherapy }\end{array}$ & FACT-B \\
\hline $\begin{array}{l}\text { Alawadi S [25], } \\
\text { Kuwait-2009 }\end{array}$ & Cross-sectional & 348 & $48.3(10.3)$ & $\begin{array}{c}\text { I: } 7 \\
\text { II: } 34.3 \\
\text { III: } 36 \\
\text { IV: } 22.7 \\
\end{array}$ & $\begin{array}{l}\text { Chemotherapy: } 98.3 \\
\text { Radiotherapy: } 21 \\
\text { Surgery: } 58.6\end{array}$ & During chemotherapy & $\begin{array}{l}\text { EORTC-QLQ-C30 } \\
\text { plus QLQ-BR23 }\end{array}$ \\
\hline $\begin{array}{l}\text { Albabtain H [26], } \\
\text { Saudi } \\
\text { Arabia-2018 }\end{array}$ & Cross-sectional & 95 & $\begin{array}{c}\text { 25-39 years: } 26.3 \\
40-59 \text { years: } 47.4 \\
\text { > } 60 \text { years: } 26.3\end{array}$ & - & $\begin{array}{l}89.5 \text { undergoing cancer } \\
\text { treatment }\end{array}$ & During cancer treatment & $\begin{array}{l}\text { EORTC-QLQ-C30 } \\
\text { plus QLQ-BR23 }\end{array}$ \\
\hline $\begin{array}{l}\text { Almutairi K [27], } \\
\text { Saudi } \\
\text { Arabia-2016 }\end{array}$ & Cross-sectional & 145 & $\begin{array}{l}\text { 26-35 years: } 13.1 \\
\text { 36-45 years: } 30.3 \\
\text { >46 years: } 56.6\end{array}$ & $\begin{array}{c}\text { I: } 21.4 \\
\text { II: } 57.9 \\
\text { III and IV: } 20 \\
\text { Distant metastasis: } 0.7\end{array}$ & $\begin{array}{c}\text { Chemotherapy: } 3.4 \\
\text { Radiotherapy: } 2.1 \\
\text { Surgery: } 31.7 \\
\text { Hormone therapy: } 0.7 \\
\text { Combination modalities: } 62.1\end{array}$ & $\begin{array}{l}\text { During the visit of } \\
\text { outpatient clinics }\end{array}$ & $\begin{array}{l}\text { EORTC-QLQ-C30 } \\
\text { plus QLQ-BR23 }\end{array}$ \\
\hline $\begin{array}{l}\text { Al-Naggar R [28], } \\
\text { Yemen-2011 }\end{array}$ & Cross-sectional & 106 & $<55$ years: 67.9 & III: 35.8 & $\begin{array}{c}\text { Chemotherapy: } 94.3 \\
\text { Radiotherapy: } 63.2 \\
\text { Surgery: } 85.8\end{array}$ & unspecified & FACT-B \\
\hline
\end{tabular}




\begin{tabular}{|c|c|c|c|c|c|c|c|}
\hline \multirow{2}{*}{$\begin{array}{l}\text { Author Name, } \\
\text { Country-Year of } \\
\text { Publication }\end{array}$} & \multirow[t]{2}{*}{ Type of Study } & \multirow[t]{2}{*}{ Sample Size } & \multirow{2}{*}{$\begin{array}{c}\begin{array}{c}\text { Age of BC } \\
\text { Patients }\end{array} \\
\text { Mean (SD) } \\
\end{array}$} & \multirow{2}{*}{$\begin{array}{c}\text { Stage } \\
\%\end{array}$} & \multirow{2}{*}{$\begin{array}{c}\text { Treatment } \\
\%\end{array}$} & \multirow[t]{2}{*}{ Time of Assessment } & \multirow[t]{2}{*}{ Questionnaires } \\
\hline & & & & & & & \\
\hline $\begin{array}{l}\text { Al-Natour A [29], } \\
\text { Jordan-2017 }\end{array}$ & Cross-sectional & 150 & $47.9(9.7)$ & $\begin{array}{l}\text { I: } 25.5 \\
\text { II: } 48.9 \\
\text { III: } 19 \\
\text { IV: } 6.6\end{array}$ & - & During cancer treatment & FACT-G \\
\hline $\begin{array}{l}\text { Bagheri M [30], } \\
\text { Iran-2015 }\end{array}$ & Case- Control & $\begin{array}{c}50 \text { cases } \\
50 \text { Healthy control }\end{array}$ & $\begin{array}{c}\text { Cases: } 32(0.5) \\
\text { Controls: } 33.7(4.2)\end{array}$ & - & - & unspecified & SF-36 \\
\hline $\begin{array}{l}\text { Bayram Z [31], } \\
\text { Turkey—2014 }\end{array}$ & Cross-sectional & 105 & $50.1(11.8)$ & $\begin{array}{c}\text { I: } 40 \\
\text { II: } 37.1 \\
\text { III: } 19.0 \\
\text { IV: } 3.8 \\
\end{array}$ & Chemotherapy: 100 & $\begin{array}{l}\text { Newly diagnosed and } \\
\text { undergoing } \\
\text { chemotherapy }\end{array}$ & FACT-G \\
\hline $\begin{array}{l}\text { Filazoglu G [32], } \\
\text { Turkey-2008 }\end{array}$ & Cross-sectional & 188 & $45.1(5.6)$ & $\begin{array}{l}\text { I: } 23.4 \\
\text { II: } 55.9 \\
\text { III: } 18.6 \\
\text { IV: } 2.1\end{array}$ & $\begin{array}{c}\text { Chemotherapy: } 17.6 \\
\text { Radiotherapy: } 38.8 \\
\text { Chemotherapy + Radiotherapy: } \\
14.4 \\
\text { No treatment: } 29.3 \\
\text { Surgery: } 100\end{array}$ & $\begin{array}{l}\text { After breast surgery for } \\
\text { a minimum of } 3 \text { months } \\
\text { before the study. }\end{array}$ & SF-36 \\
\hline $\begin{array}{l}\text { Hujeir H [33], } \\
\text { Lebanon-2012 }\end{array}$ & Cross-sectional & 89 & $49.2(11.1)$ & Metastasis: 37.1 & $\begin{array}{l}\text { Chemotherapy: } 47.2 \\
\text { Radiotherapy: } 31.4 \\
\text { Surgery: } 70.7 \\
\text { Hormone therapy: } 15.7 \\
\end{array}$ & $\begin{array}{l}30.6(39.06) \\
\text { months }\end{array}$ & EORTC-QLQ-C30 \\
\hline $\begin{array}{l}\text { Jafari N [34], } \\
\text { Iran-2013 }\end{array}$ & Cross-sectional & 68 & $48(10.3)$ & - & Radiotherapy: 100 & During Radiotherapy & $\begin{array}{l}\text { EORTC-QLQ-C30 } \\
\text { plus QLQ-BR23 }\end{array}$ \\
\hline $\begin{array}{l}\text { Jassim G [35], } \\
\text { Bahrain—2013 }\end{array}$ & Cross-sectional & 239 & $50.2(11.1)$ & $\begin{array}{c}\text { I: } 29.9 \\
\text { II: } 44.8 \\
\text { III and IV: } 25.3\end{array}$ & $\begin{array}{l}\text { Chemotherapy: } 80.5 \\
\text { Radiotherapy: } 83.9 \\
\text { Lumpectomy: } 51.3 \\
\text { Mastectomy: } 50 \\
\text { Lymph node dissection: } 85.1 \\
\text { Hormone therapy: } 69.8\end{array}$ & $\begin{array}{c}\text { Early diagnosed: } 14.6 \\
\text { Transitional period: } 53.6 \\
\text { Long-term survivors: } \\
31.8\end{array}$ & $\begin{array}{l}\text { EORTC-QLQ-C30 } \\
\text { plus QLQ-BR23 }\end{array}$ \\
\hline $\begin{array}{l}\text { Khalili N [36], } \\
\text { Iran-2013 }\end{array}$ & Cross-sectional & 62 & $45.8(6.7)$ & $\begin{array}{l}\text { IA: } 1.6 \\
\text { IB: } 6.4 \\
\text { IIA: } 22.5 \\
\text { IIB: } 25.8 \\
\text { IIIA: } 16.1 \\
\text { IIIB: } 14.5 \\
\text { IIIC: } 8.1\end{array}$ & - & $\begin{array}{l}\text { Diagnosis with } B C \text { in a } \\
\text { recent year }\end{array}$ & EORTC-QLQ-C30 \\
\hline
\end{tabular}




\begin{tabular}{|c|c|c|c|c|c|c|c|}
\hline \multirow{2}{*}{$\begin{array}{l}\text { Author Name, } \\
\text { Country-Year of } \\
\text { Publication }\end{array}$} & \multirow[t]{2}{*}{ Type of Study } & \multirow[t]{2}{*}{ Sample Size } & \multirow{2}{*}{$\begin{array}{c}\begin{array}{c}\text { Age of BC } \\
\text { Patients }\end{array} \\
\text { Mean (SD) } \\
\end{array}$} & \multirow{2}{*}{$\begin{array}{c}\text { Stage } \\
\%\end{array}$} & \multirow{2}{*}{$\begin{array}{c}\text { Treatment } \\
\%\end{array}$} & \multirow[t]{2}{*}{ Time of Assessment } & \multirow[t]{2}{*}{ Questionnaires } \\
\hline & & & & & & & \\
\hline $\begin{array}{l}\text { Kiadaliri A [37], } \\
\text { Iran-2012 }\end{array}$ & Longitudinal & 100 & $\begin{array}{l}\text { TAC: } 46.7(8.2) \\
\text { FAC: } 49.3(11.5)\end{array}$ & - & - & $\begin{array}{c}\text { Before and after } \\
\text { chemotherapy and } 4 \\
\text { months later }\end{array}$ & EORTC-QLQ-C30 \\
\hline $\begin{array}{l}\text { Mohammadi S } \\
\text { [38], Iran-2013 }\end{array}$ & Cross-sectional & 100 & $47.8(6.7)$ & $\begin{array}{l}\text { I: } 8 \\
\text { II: } 41 \\
\text { III: } 51.3\end{array}$ & $\begin{array}{l}\text { All patients completed } 3 \text { phases } \\
\text { of BC treatment, which } \\
\text { included mastectomy, } \\
\text { chemotherapy, radiation } \\
\text { therapy. }\end{array}$ & $\begin{array}{l}\text { Duration of } \\
\text { survivorship: } \\
2 \text { years: } 25 \\
3 \text { years: } 44 \\
4 \text { years: } 25 \\
5 \text { years: } 6\end{array}$ & EORTC-QLQ-C30 \\
\hline $\begin{array}{l}\text { Moradi R [39], } \\
\text { Iran-2017 }\end{array}$ & Cross-sectional & 87 & 48.25 (11.9) & - & Chemotherapy: 100 & After chemotherapy & WHOQOL-BREF \\
\hline $\begin{array}{l}\text { Musarezaei A [40], } \\
\text { Iran-2015 }\end{array}$ & Cross-sectional & 105 & $45.3(4.6)$ & II: 50 & Mastectomy: 100 & $\begin{array}{l}\text { Underwent mastectomy } \\
\text { (at least } 1 \text { year and a } \\
\text { maximum of } 5 \text { years } \\
\text { previously) }\end{array}$ & NMCBRI-Q \\
\hline $\begin{array}{l}\text { Najafi F [41], } \\
\text { Iran-2016 }\end{array}$ & Cross-sectional & 148 & $47.6(10.1)$ & $\begin{array}{l}\text { In situ: } 7.1 \\
\text { Local: } 45.7 \\
\text { Loco/regional: } 40 \\
\text { Advanced: } 7.1\end{array}$ & $\begin{array}{l}\text { Patients completed at least } 2 \\
\text { chemotherapy sessions (only } \\
\text { those with very small tumors } \\
\text { did not undergo } \\
\text { chemotherapy). }\end{array}$ & $\begin{array}{l}\text { Time since diagnosis: } \\
17.2 \text { (1.4) months }\end{array}$ & EORTC-QLQ-C30 \\
\hline $\begin{array}{l}\text { Nikamenesh Z } \\
\text { [42], Iran-2017 }\end{array}$ & Cross-sectional & 42 & $30-50$ years: $69 \%$ & - & - & $\begin{array}{c}\text { Diagnosis for at least } 6 \\
\text { months and under } \\
\text { treatment }\end{array}$ & EORTC-QLQ-C30 \\
\hline $\begin{array}{l}\text { Pehlivan S [43], } \\
\text { Turkey-2016 }\end{array}$ & Cross-sectional & 61 & $50.37(11.8)$ & $\begin{array}{l}\text { I: } 9.8 \\
\text { II: } 59 \\
\text { III: } 23 \\
\text { IV: } 8.2\end{array}$ & $\begin{array}{c}\text { Chemotherapy: } 19.7 \\
\text { Chemotherapy + Radiotherapy: } \\
80.3\end{array}$ & During Radiotherapy & $\begin{array}{c}\text { EORTC } \\
\text { QLQ-BR23 }\end{array}$ \\
\hline $\begin{array}{l}\text { Rohani C [44], } \\
\text { Iran-2015 }\end{array}$ & Case-control & $\begin{array}{l}\text { Cases (BC } \\
\text { patients): } 162 \\
\text { Controls: } 210\end{array}$ & $\begin{array}{c}\text { Cases: } 46.1(9.8) \\
\text { Control: } 46.6(8.4)\end{array}$ & $\begin{array}{c}0: 2.5 \\
\text { I: } 22.8 \\
\text { II: } 49.4 \\
\text { III: } 24.1 \\
\text { IV: } 1.2\end{array}$ & $\begin{array}{l}\text { Chemotherapy: } 79 \\
\text { Radiotherapy: } 75.3 \\
\text { Hormone therapy: } 68.5\end{array}$ & $\begin{array}{c}\text { Baseline pre-diagnosis } \\
\text { phase of } \mathrm{BC}(\mathrm{T} 1) \text { and } 6 \\
\text { months post } \\
\text { pre-diagnosis (T2). }\end{array}$ & EORTC-QLQ-C30 \\
\hline $\begin{array}{l}\text { Safa A [45], } \\
\text { Iran-2014 }\end{array}$ & Cross-sectional & 92 & 42.9 (8.7) & - & - & $\begin{array}{c}\text { At least } 3 \text { months since } \\
\text { treatment }\end{array}$ & QOL-BC \\
\hline
\end{tabular}




\begin{tabular}{|c|c|c|c|c|c|c|c|}
\hline \multirow{2}{*}{$\begin{array}{l}\text { Author Name, } \\
\text { Country-Year of } \\
\text { Publication }\end{array}$} & \multirow[t]{2}{*}{ Type of Study } & \multirow[t]{2}{*}{ Sample Size } & $\begin{array}{l}\text { Age of BC } \\
\text { Patients }\end{array}$ & Stage & Treatment & \multirow[t]{2}{*}{ Time of Assessment } & \multirow[t]{2}{*}{ Questionnaires } \\
\hline & & & Mean (SD) & $\%$ & $\%$ & & \\
\hline $\begin{array}{l}\text { Shakeri J [46], } \\
\text { Iran-2016 }\end{array}$ & Cross-sectional & 98 & $47.6(14)$ & - & - & Unspecified & LQI \\
\hline $\begin{array}{l}\text { Saatci E [47], } \\
\text { Turkey-2007 }\end{array}$ & Cross-sectional & 100 & 48.64 (10.6) & $\begin{array}{l}\text { I: } 49 \\
\text { II: } 51\end{array}$ & $\begin{array}{c}\text { Chemotherapy: } 59 \\
\text { Radiotherapy: } 10 \\
\text { Chemotherapy + Radiotherapy: } \\
31\end{array}$ & 22.6 (24.3) months & FACT-G \\
\hline $\begin{array}{l}\text { Safaee A [48], } \\
\text { Iran-2008 }\end{array}$ & Cross-sectional & 119 & 48.27 (11.4) & $\begin{array}{c}\text { Well differentiated: } \\
33.6 \\
\text { Moderately } \\
\text { differentiated: } 42 \\
\text { Poorly differentiated: } \\
24.4\end{array}$ & Chemotherapy: 100 & $\begin{array}{l}\text { Time since diagnosis: } \\
\text { < } 4 \text { months: } 39.5 \\
4-12 \text { months: } 34.4 \\
\text { >12 months: } 26.1\end{array}$ & EORTC-QLQ-C30 \\
\hline $\begin{array}{l}\text { Shandiz,F H [49], } \\
\text { Iran-2008 }\end{array}$ & Cross-sectional & 94 & $45.20(8.6)$ & - & $\begin{array}{c}\text { Chemotherapy: } 100 \\
\text { Radiotherapy: } 69.1 \\
\text { Surgery: } 100 \\
\text { Hormone therapy: } 52.1 \\
\end{array}$ & During chemotherapy & EORTC-QLQ-C30 \\
\hline $\begin{array}{l}\text { Sinaei F [50], } \\
\text { Iran-2017 }\end{array}$ & Case-control & $\begin{array}{l}\text { Mastectomy } \\
\text { group: } 45 \\
\text { Reconstruction } \\
\text { group: } 61\end{array}$ & $\begin{array}{l}\text { Mastectomy: } \\
50.2(8.5) \\
\text { Reconstruction: } \\
46.7(8.1)\end{array}$ & - & $\begin{array}{c}\text { Surgery: } 100 \\
\text { Chemo radiation: } \\
\text { Mastectomy: } 86.7 \\
\text { Reconstruction: } 90 \\
\end{array}$ & unspecified & $\begin{array}{l}\text { EORTC-QLQ-C30 } \\
\text { plus QLQ-BR23 }\end{array}$ \\
\hline $\begin{array}{l}\text { Uzun Ö [51], } \\
\text { Turkey-2004 }\end{array}$ & Cross-sectional & 72 & $50.1(9.3)$ & $\begin{array}{l}\text { I: } 24 \\
\text { II: } 58.3 \\
\text { III: } 16.7\end{array}$ & $\begin{array}{l}\text { Chemotherapy: } 100 \\
\text { Radiotherapy: } 52.8 \\
\text { Surgery: } 100\end{array}$ & unspecified & QoLS \\
\hline $\begin{array}{l}\text { Zamanian H [52], } \\
\quad \text { Iran-2015 }\end{array}$ & Cross-sectional & 224 & $47.1(9.0)$ & - & $\begin{array}{l}\text { Chemotherapy: } 61.6 \\
\text { Radiotherapy: } 40 \\
\text { Surgery: } 26.8\end{array}$ & $\begin{array}{l}\text { Time since diagnosis: } \\
43.8 \text { (37.6) months }\end{array}$ & FACT-B \\
\hline $\begin{array}{l}\text { Zargani A [53], } \\
\text { Iran-2018 }\end{array}$ & Cross-sectional & 84 & $54.7(10.4)$ & - & $\begin{array}{l}\text { All patients undergoing cancer } \\
\text { treatment (surgery, } \\
\text { radiotherapy or chemotherapy). }\end{array}$ & $\begin{array}{l}\text { Diagnosis at least } 1 \text { year } \\
\text { prior }\end{array}$ & SF-36 \\
\hline $\begin{array}{l}\text { Age of breast canc } \\
\text { surgery, and horm } \\
\text { and treatment of } ~ \\
\text { Therapy-General } \\
\text { SF-36: Short-Form } \\
\text { doxorubicin, and c }\end{array}$ & $\begin{array}{l}\text { atients and time } \\
\text { otherapy) are pr } \\
\text { cer-Quality of } \\
\text { eestionnaire. LQ } \\
\text { ealth Survey Qu } \\
\text { ophosphamide. }\end{array}$ & $\begin{array}{l}\text { diagnosis of } \\
\text { d as number } \\
\text { Questionnaire. } \\
\text { quality invent } \\
\text { inaire. WHOC }\end{array}$ & $\begin{array}{l}\text { presented as } \\
\text { ntage). Abbre } \\
\text { T-B: The Func } \\
\text { JMCBRI-Q: Th } \\
\text { REF: WHO qu }\end{array}$ & $\begin{array}{l}\text { (standard deviation } \\
\text { ns: BR23: Breast can } \\
\text { Assessment of Can } \\
\text { ional medical center } \\
\text { nnaire of quality of }\end{array}$ & $\begin{array}{l}\text {, stage of BC I, II, III, and IV, an } \\
\text { er specific module. EORTC-QL } \\
\text { er Therapy-Breast Cancer. FA } \\
\text { nd Beckman research institute } \mathrm{q} \\
\text { fe. FAC: fluorouracil, doxorubi }\end{array}$ & $\begin{array}{l}\text { interventions (chemoth } \\
\text { 2-C30: European Organ } \\
\text { TT-G: The Functional As } \\
\text { lestionnaire. QoLS: The } \\
\text { in, and cyclophosphami }\end{array}$ & $\begin{array}{l}\text { apy, radiotherapy, } \\
\text { ation for Research } \\
\text { essment of Cancer } \\
\text { uality of Life Scale. } \\
\text { e. TAC: docetaxel, }\end{array}$ \\
\hline
\end{tabular}




\subsubsection{Characteristics of the Cross-Sectional Studies}

Sample sizes of the included cross-sectional studies $(n=28)$ ranged from 42 to 762 patients, and totaled 8764 women with BC included in all studies. Age ranged from 25 to 60 years. The studies included were published between 2004 and 2018. For outcome assessment, specific BC questionnaires, such as the European Organization for Research and treatment of Cancer-Quality of Life Questionnaire (EORTC QLQ-C30) associated with the breast cancer module (QLQ-BR23) $(n=8)$, the Functional Assessment of Cancer Therapy-Breast Cancer (FACT-B) $(n=4)$, as well as generic tools were used.

\subsubsection{Characteristics of the Case-Control Studies}

The total number of women with BC in case-control studies was 318, with a mean age ranging from 32 to 49 years. These studies were published between 2015 and 2017, and were reported from Iran. One study used the EORTC QLQ-C30, the second one used the EORTC QLQ-C30 associated with the QLQ-BR23, and the third used the generic SF-36 questionnaire.

\subsubsection{Characteristics of the Longitudinal Studies}

Concerning the two longitudinal studies, a total number of 241 BC women with mean ages of 46.9 and 47.2 years were included. The two studies were published in 2008 and were reported from Turkey and Iran. With regard to the instruments used to assess HRQoL, one study used the FACT-B and the other used the EORTC QLQ-C30.

\subsection{Quality Assessment}

A summary of the quality assessment results is presented in Table $4 \mathrm{a}, \mathrm{b}$. According to quality criteria on the NOS scale, ten cross-sectional studies were classed as high quality (five stars or more out of six). Two case-control studies and one longitudinal study were of high quality (six stars out of nine).

Table 4. Newcastle-Ottawa scale for the assessment of the quality of studies included. (a). Quality assessment of cross-sectional studies and longitudinal studies according to the Newcastle-Ottawa scale. (b). Newcastle-Ottawa scale for the assessment of the quality of case-control studies (each asterisk indicates if the individual criterion within the subsection was fulfilled).

\begin{tabular}{|c|c|c|c|c|c|}
\hline Author & Study Design & $\begin{array}{l}\text { Selection of } \\
\text { Participants }\end{array}$ & $\begin{array}{l}\text { Comparability } \\
\text { Based on Design } \\
\text { or Analysis }\end{array}$ & $\begin{array}{l}\text { Assessment of } \\
\text { Outcome/Exposure }\end{array}$ & Total \\
\hline \multicolumn{6}{|c|}{ (a) } \\
\hline Ahmed et al. [21] & Cross-sectional & $* *$ & $* *$ & $*$ & $5(/ 6)$ \\
\hline Ahrafizadeh et al. [22] & Cross-sectional & $* *$ & & * & $3(/ 6)$ \\
\hline Akel et al. [23] & Cross-sectional & $* *$ & & * & $3(/ 6)$ \\
\hline Akin et al. [24] & longitudinal & $* * *$ & & $* *$ & $5(/ 9)$ \\
\hline Alawadi et al. [25] & Cross-sectional & $* *$ & $* *$ & * & $5(/ 6)$ \\
\hline Albabtain et al. [26] & Cross-sectional & $* *$ & & * & $3(/ 6)$ \\
\hline Almutairi et al. [27] & Cross-sectional & $* *$ & $* *$ & * & $5(/ 6)$ \\
\hline Al Naggar et al. [28] & Cross-sectional & $* *$ & $* *$ & * & $5(/ 6)$ \\
\hline Al Natour et al. [29] & Cross-sectional & $* *$ & & * & $3(/ 6)$ \\
\hline Bayram et al. [31] & Cross-sectional & $* *$ & & * & $3(/ 6)$ \\
\hline Filazoglu et al. [32] & Cross-sectional & $* *$ & $* *$ & $*$ & $5(/ 6)$ \\
\hline Hujeir et al. [33] & Cross-sectional & $* *$ & & * & $3(/ 6)$ \\
\hline Jafari et al. [34] & Cross-sectional & $* *$ & * & * & $4(/ 6)$ \\
\hline Jassim et al. [35] & Cross-sectional & $* *$ & $* *$ & $*$ & $5(/ 6)$ \\
\hline Khalili et al. [36] & Cross-sectional & $* *$ & & $*$ & $3(/ 6)$ \\
\hline Kiadaliri et al. [37] & longitudinal & $* * *$ & & $* *$ & $5(/ 9)$ \\
\hline Mohammadi et al. [38] & Cross-sectional & $* *$ & & $*$ & $3(/ 6)$ \\
\hline
\end{tabular}




\begin{tabular}{|c|c|c|c|c|c|}
\hline Author & Study Design & $\begin{array}{l}\text { Selection of } \\
\text { Participants }\end{array}$ & $\begin{array}{c}\text { Comparability } \\
\text { Based on Design } \\
\text { or Analysis }\end{array}$ & $\begin{array}{l}\text { Assessment of } \\
\text { Outcome/Exposure }\end{array}$ & Total \\
\hline Moradi et al. [39] & Cross-sectional & $* * *$ & & * & $4(/ 6)$ \\
\hline Musarezaei et al. [40] & Cross-sectional & ** & & * & $3(/ 6)$ \\
\hline Najafi et al. [41] & Cross-sectional & $* * *$ & $* *$ & * & $6(/ 6)$ \\
\hline Nikmanesh et al. [42] & Cross-sectional & $* *$ & & * & $3(/ 6)$ \\
\hline Pehlivan et al. [43] & Cross-sectional & ** & & * & $3(/ 6)$ \\
\hline Saatci et al. [47] & Cross-sectional & $* *$ & & $*$ & $3(/ 6)$ \\
\hline Safa et al. [45] & Cross-sectional & $* * *$ & & * & $4(/ 6)$ \\
\hline Safaee et al. [48] & Cross-sectional & $* *$ & $* *$ & * & $5(/ 6)$ \\
\hline Shakeri et al. [46] & Cross-sectional & $* *$ & & * & $3(/ 6)$ \\
\hline Shandiz et al. [49] & Cross-sectional & $* *$ & $* *$ & * & $5(/ 6)$ \\
\hline Uzun et al. [51] & Cross-sectional & $* *$ & & * & $3(/ 6)$ \\
\hline Zamanian et al. [52] & Cross-sectional & $* *$ & $* *$ & * & $5(/ 6)$ \\
\hline Zargani et al. [53] & Cross-sectional & $* *$ & & * & $3(/ 6)$ \\
\hline \multicolumn{6}{|c|}{ (b) } \\
\hline Bagheri et al. [30] & Case-control & * & * & $* *$ & $4(/ 9)$ \\
\hline Rohani et al. [44] & Case-control & $* * *$ & $* *$ & $* *$ & $7(/ 9)$ \\
\hline Sinaei et al. [50] & Case-control & $* * *$ & ** & ** & $7(/ 9)$ \\
\hline
\end{tabular}

"*" star system: for cohort study: A study can be awarded a maximum of one star for each numbered item within the Selection and Outcome categories. A maximum of two stars can be given for Comparability. For cross-sectional study: A study can be awarded a maximum of one star for each numbered item within the Selection and Exposure categories. A maximum of two stars can be given for Comparability.

\subsection{Factors Associated with HRQoL in Women with BC in the Middle East}

The authors of the included studies identified several factors associated with global HRQOL in women with BC. Table 5 presents a summary of these factors.

Table 5. Global health-related quality of life (HRQoL) and its associated factors in women with breast cancer in the Middle East.

\begin{tabular}{|c|c|c|c|}
\hline Author Name & Global HRQoL Mean (SD) & Determinants of HRQoL & $\begin{array}{l}\text { Signs of } \\
\text { Association }\end{array}$ \\
\hline \multirow{5}{*}{ Ahmed A et al. [21] } & \multirow{5}{*}{$50.7(19.2)$} & Regular exercise & + \\
\hline & & Multiple tumors & - \\
\hline & & Metastasis & - \\
\hline & & Fever & - \\
\hline & & Age & - \\
\hline \multirow{4}{*}{ Ahrafizadeh H [22] } & \multirow{3}{*}{$87.9(2.15)$} & Higher level of education & + \\
\hline & & Higher income & + \\
\hline & & $\begin{array}{c}\text { Type of surgery (breast conserving surgery } \\
\text { versus mastectomy) }\end{array}$ & + \\
\hline & \multirow{6}{*}{$108.7(18.7)$} & Duration of the disease/diagnosis & + \\
\hline \multirow{5}{*}{ Akel R [23] } & & Iraqi & - \\
\hline & & Stage IV & - \\
\hline & & Monthly income below 1000 USD & - \\
\hline & & Chemotherapy & - \\
\hline & & Anxiety and depression & - \\
\hline \multirow{9}{*}{ Akin S [24] } & \multirow{9}{*}{$\begin{array}{l}\text { Before chemotherapy: } 69.2(21.6) \\
\text { chemotherapy: } 53.3(20.5)\end{array}$} & Age (40-50 years) & - \\
\hline & & Married & - \\
\hline & & Employed women & - \\
\hline & & Low income & - \\
\hline & & Obesity & - \\
\hline & & Stage I, II, III, & - \\
\hline & & FAC/FEC and Docetaxel/Paclitaxel AC/EC & - \\
\hline & & Diagnosis since $2-6$ months & - \\
\hline & & Breast-sparing surgical procedure & - \\
\hline
\end{tabular}




\begin{tabular}{|c|c|c|c|}
\hline Author Name & Global HRQoL Mean (SD) & Determinants of HRQoL & $\begin{array}{c}\text { Signs of } \\
\text { Association }\end{array}$ \\
\hline \multirow{4}{*}{ Alawadi S [25] } & \multirow{4}{*}{$45.3(15.3)$} & Social functioning & - \\
\hline & & Sexual enjoyment & - \\
\hline & & Pain & - \\
\hline & & Financial difficulty & + \\
\hline Albabtain H [26] & \multirow{2}{*}{$\begin{array}{c}\text { CAM }=73.1(20.2) \\
\text { No CAM }=64.8(32.7) \\
31.2(20.5)\end{array}$} & Complementary alternative medicine & + \\
\hline Almutairi K [27] & & Pathological staging & - \\
\hline \multirow{3}{*}{ Al-Naggar R [28] } & \multirow{3}{*}{$\begin{array}{l}<55 \text { years }=83.2(22.8) \\
\geq 55 \text { years }=77(21.6)\end{array}$} & Lower income & - \\
\hline & & $>2$ years after diagnosis & - \\
\hline & & Under radiotherapy & + \\
\hline Al-Natour A [29] & $79.9(18.1)$ & Spiritual wellbeing & + \\
\hline Bagheri M [30] & $73.3(11.2)$ & $\begin{array}{l}\text { Body image, Appearance evaluative, } \\
\text { Appearance orientation, Fitness evaluative, } \\
\text { Fitness orientation, Subjective weight, Body } \\
\text { satisfaction }\end{array}$ & + \\
\hline \multirow[t]{2}{*}{ Bayram Z [31] } & $63.8(16.4)$ & $\begin{array}{l}\text { Literate, Homemakers, ECOG2, Stage IV, } \\
\text { Chemotherapy agents } \\
\text { (gemcitabine+cisplatin). }\end{array}$ & - \\
\hline & \multirow{13}{*}{-} & Physical component score: & PCS: \\
\hline \multirow{12}{*}{ Filazoglu G [32] } & & Stage of Cancer & - \\
\hline & & Time since operation & + \\
\hline & & Time since diagnosis & + \\
\hline & & Social support & + \\
\hline & & Problem solving coping & + \\
\hline & & Mental component score: & MCS: \\
\hline & & Type of treatment & - \\
\hline & & Stage of Cancer & - \\
\hline & & Time since operation & + \\
\hline & & Social support & + \\
\hline & & Problem solving coping & + \\
\hline & & Helplessness coping & - \\
\hline \multirow{4}{*}{ Hujeir H [33] } & \multirow{4}{*}{$59.6(29.0)$} & Psychological symptoms & - \\
\hline & & Physical symptoms & - \\
\hline & & Total memorial symptom assessment scale & - \\
\hline & & Social functioning & + \\
\hline \multirow{3}{*}{ Jafari N [34] } & \multirow{3}{*}{$41.4(18.0)$} & Pain & - \\
\hline & & Arm symptoms & - \\
\hline & & Spiritual well-being & + \\
\hline Jassim G [35] & $63.9(21.3)$ & Advanced stage & - \\
\hline Jassint u [30] & (2) & Menopause & - \\
\hline Khalili N [36] & $603(2110)$ & Emotion focused coping strategy & + \\
\hline Knallm N [36] & $60.3(21.10)$ & Affective interference of pain & - \\
\hline & Baseline: & Having children & + \\
\hline Kiadaliri A [37] & TAC: $69.3(0.9)$ & Being married & - \\
\hline & FAC: $69.4(1.5)$ & TAC treatment & - \\
\hline Mohammadi S [38] & $68.1(17.7)$ & Nutritional status (well-nourished) & + \\
\hline Moradi R [39] & $75.9(15.2)$ & Economic status & + \\
\hline & Weak HRQoL $16.36 \%$ & Self-efficacy & + \\
\hline Musarezaei A [40] & $\begin{array}{l}\text { Moderate HRQoL 58.48\% } \\
\text { Good HRQoL 25.15\% }\end{array}$ & Higher level of education & + \\
\hline & & Fatigue & - \\
\hline Najafi F [41] & $43.86(23.5)$ & Older Age & + \\
\hline Nikamenesh Z [42] & $10.78(2.4)$ & Self-regulation & + \\
\hline Pehlivan S [43] & $55.1(10.8)$ & Age, RTCS, Functional, Symptom & + \\
\hline & Baseline: & & \\
\hline & BC group: 58.1 (20.1) & Sense of coherence & + \\
\hline Rohani C [44] & Control group: 70.1(21.6) & & \\
\hline Konani C [44] & 6 months post pre-diagnosis: & & \\
\hline & BC group: 68.7 (18.5) & Education & + \\
\hline & Control group: 72.4 (18) & & \\
\hline & & Age & + \\
\hline Safa A [45] & Average QoL: 87\% & Disease duration & + \\
\hline & High QoL: 13\% & Fatigue & + \\
\hline & & Interval between diagnosis and treatment & - \\
\hline
\end{tabular}




\begin{tabular}{|c|c|c|c|}
\hline Author Name & Global HRQoL Mean (SD) & Determinants of HRQoL & $\begin{array}{l}\text { Signs of } \\
\text { Association }\end{array}$ \\
\hline Shakeri J [46] & $70.02(14.4)$ & Depression & - \\
\hline \multirow{4}{*}{ Saatci E [47] } & \multirow{4}{*}{$76.1(14.8)$} & Unmet needs & + \\
\hline & & Working women & + \\
\hline & & Grade of tumor & - \\
\hline & & Occupation & + \\
\hline \multirow[t]{5}{*}{ Safaee A [48] } & \multirow[t]{5}{*}{$64.9(11.4)$} & Post menopause & + \\
\hline & & Financial difficulties & - \\
\hline & & Dyspnea & - \\
\hline & & Older age & - \\
\hline & & High social status & + \\
\hline \multirow{4}{*}{ Shandiz,F H [49] } & \multirow{4}{*}{$71.4(22.3)$} & No radiotherapy & + \\
\hline & & Receiving hormone therapy & + \\
\hline & & Fatigue & - \\
\hline & & Dyspnea & - \\
\hline Sinaei F [50] & $\begin{array}{c}\text { Mastectomy group: } 57.1 \\
\text { Reconstruction group: } 72.5\end{array}$ & $\begin{array}{l}\text { Breast reconstruction surgery more positive } \\
\text { effect on QoL than Mastectomy }\end{array}$ & + \\
\hline \multirow{2}{*}{ Uzun Ö [51] } & \multirow{2}{*}{$147.6(24.0)$} & Education & + \\
\hline & & Employed women & + \\
\hline Zamanian H [52] & $105.0(22.7)$ & Positive religious coping & + \\
\hline Zargani A [53] & $73.1(9.2)$ & Religiosity & + \\
\hline
\end{tabular}

Abbreviations: AC: Doxorubicin/Cyclophosphamide. CAM: Complementary alternative medicine. EC: Epirubicin/Cyclophosphamide. ECOG: Eastern cooperative oncology group performance score. FAC = 5-fluorouracil, doxorubicin, and cyclophosphamide. FEC: Fluorouracil, Epirubicin, Cyclophosphamide. RTCS: Radiation therapy comfort scale. TAC: Docetaxel with doxorubicin and cyclophosphamide. +: signifies a positive association between determinants and HRQoL, determinant develop a better HRQoL.: signifies a negative association between determinants and HRQoL, determinant develop a lower HRQoL.

\subsubsection{Socio-Demographic Factors}

Several socio-demographic factors were closely associated with HRQoL in patients with BC. These included: age [21,24,41,43,45,49], marital status [24,37], level of education [22,31,40,44,51], employment status [47,48,51], income [22-25,28,39,48], and having children [37]. Regarding age, the direction of the association was not consistent across studies, with three studies reporting a negative association with HRQoL [21,24,49], while three other studies reported the opposite [41,43,45]. Being employed, having a higher level of education, and having children were factors found to be associated with better HRQoL. Conversely, being married, having a low income, and financial difficulties adversely affected HRQoL.

\subsubsection{Clinical Factors}

Multiple clinical factors, including multiple tumors [21], cancer stage $[23,24,27,31,32,35,48]$, metastasis [21], time since diagnosis [24,28,32,45], time since operation [32], disease duration [45], menopausal status [35,48], higher symptom score on the Memorial Symptom Assessment Scale [33], Eastern cooperative oncology group performance score [30], fever [21], pain [25,34,36], fatigue [40,49], and dyspnea $[48,49]$ were found to be associated with HRQoL.

\subsubsection{Treatment-Related Factors}

HRQoL was negatively influenced by chemotherapy, including Docetaxel with doxorubicin and cyclophosphamide/Gemcitabine + cisplatin/FAC/FEC and Docetaxel/Paclitaxel AC/EC in four studies [23,24,31,37] and by breast-sparing surgery in one study [24], whereas other studies reported that HRQoL was positively influenced by hormone therapy [49], early treatment [45], and breast reconstruction surgery [50]. One study found a positive association with radiotherapy [28], while another reported the reverse [49]. HRQoL was positively affected by the use of complementary alternative medicine [26]. 


\subsubsection{Behavioral Factors}

Behavioral factors such as physical activity [21], fitness orientation and evaluation [30], body weight [24,29], and nutritional status [38] were reportedly associated with HRQoL, with women of normal weight and exercising regularly, and well-nourished women having better HRQoL. In addition, positive body image [30], body satisfaction [30], as well as positive religiosity [52,53] and spiritual well-being $[29,34]$ were associated with better HRQoL.

\subsubsection{Psychosocial Factors}

Several psychosocial factors were reported to have a significant impact on HRQoL. Depression [23,46], anxiety [23], psychological symptoms (including feeling nervous, feeling sad, worrying, difficulty sleeping, "I don't look like myself") [33], emotional functioning, helplessness coping [32], and unmet needs [47] had significant negative impacts on HRQoL. Conversely, self-efficacy [39], self-regulation [42], sense of coherence [44], and emotion focus coping strategy [36] had positive impacts on HRQoL.

\section{Discussion}

The main aim of the present review was to synthesize the literature exploring the factors that influence HRQoL in women with breast cancer (BC) in the Middle East. After an extensive literature review, we identified only 33 articles that met our inclusion criteria. The vast majority of these studies were cross-sectional and only three studies were longitudinal prospective studies. In almost $60 \%$, studies were published in medical journals, readily available for both medical practitioners and decision-makers. The methodological quality was high in only $39 \%$ of the studies, indicating that there was a high risk of biased results. Our comprehensive literature review identified several sociodemographic, clinical, and treatment-related factors, as well as behavioral and psychosocial factors associated with HRQoL. These findings provide a scientific basis to develop a comprehensive multidimensional program that incorporates these factors, to improve the QoL of breast cancer survivors in the Middle East.

Concerning sociodemographic factors, there was an inconsistency between studies regarding age. The results of three studies suggested that HRQoL was adversely affected in older patients, while three other studies found that younger patients with BC experienced poorer HRQoL than their older counterparts. This discrepancy may be explained by the heterogeneity of the samples, the subjects included, and a lack of power due to the low sample size. Thus, we cannot draw any clear conclusion about the effect of age on HRQoL and further studies are needed to evaluate the association between these two variables. The results of the studies reviewed here consistently suggested that highly educated woman had better quality of life compared to their less well-educated counterparts. A possible explanation for this finding is the ability of educated women to understand the nature of the disease and to comply with the therapeutic recommendations better than the less educated. Moreover, illiterate women with low income are less likely to be screened for breast cancer, delay before seeking care in the presence of symptoms, and are diagnosed at later stages of the disease. Therefore, health care teams should give more attention and support to less well-educated women (i.e., less than secondary level and illiterate individuals) with BC who need extensive information about their treatment and follow-up.

Regarding socioeconomic status, the studies in this review consistently found that patients who were unemployed and had financial difficulties or a lower monthly income reported lower HRQoL than patients who had higher incomes or no financial difficulties [54,55]. In fact, higher economic status can be linked to many aspects of improved patient care, such as rapid access to treatment and rehabilitation, as well as less concern for the financial burden of the treatment [54]. Being married was also found to be associated with better HRQoL in BC patients. In line with this finding, studies from the US and China have found that married BC patients had better QoL compared to single or divorced 
women [54,56]. This could be explained by the emotional support provided by their spouses. Finally, having children was also found to be associated with better HRQoL, although the number of studies was insufficient to draw clear conclusions.

Regarding clinical and treatment-related factors, HRQoL was significantly impaired by the type of treatment, by advanced stages of disease, and by the symptoms experienced. Chemotherapy was consistently associated with poorer HRQoL in Middle Eastern women with BC. Indeed, patients on chemotherapy are more likely to be diagnosed with advanced stage disease and to experience pain, fatigue, and possibly other severe side effects, which in turn reduce quality of life. Other treatments, such as hormone therapy and breast reconstruction surgery, are less likely to be associated with advance stage disease, and thus less likely to negatively affect quality of life. Regarding radiotherapy, findings were conflicting across studies, and no clear conclusion emerged. Interestingly, one of the studies reviewed [26] reported that complementary and alternative medicine, including spiritual therapy, honey, olive oil, and herbal therapy, was associated with better global HRQoL, physical role, and social functioning, as well as alleviating cancer-related constipation. Since this type of medicine is commonly used in the Middle East, these findings warrant confirmation with a view to incorporating them into medical care and management programs for breast cancer patients.

Concerning behavioral factors, well-nourished women [38] with normal weight [24,29] and those who exercise regularly [21] tended to have better quality of life. In line with these findings, Gong et al. reported a positive effect of physical exercise and healthy diet on HRQoL [54,56]. Thus, promoting patient participation in rehabilitation programs, including nutritional education and physical exercise, might be one way to improve HRQoL in patients with BC. Moreover, consistent with previous findings reported by Wildes et al. [57] our literature review revealed that positive religiosity [52,53] and spiritual well-being were associated with better HRQoL. However, body image disturbance and dissatisfaction were found to be associated with poor HRQoL. In fact, body image disturbance following treatment of cancer may be associated with a variety of changes, such as depression and anxiety, that can have a significant negative impact on HRQoL. Therefore, it is important to evaluate body weight perception in BC patients after chemotherapy or mastectomy, as this may affect biopsychosocial functioning [42].

Several psychosocial factors were found to be associated with HRQoL. As expected, depression and anxiety had a significant negative impact on HRQoL. This is in agreement with the findings of Poleshuck et al. and Shelby et al., who found that patients with BC may experience anxiety and depression regarding surgical experience, coping with acute pain, treatment regimens, financial burden of care, and disruptions to their personal and professional lives [58,59]. All of these factors may in turn adversely affect their quality of life. Thus, early identification and interventions to alleviate depression, anxiety, and stress may help improve HRQoL. It was also found throughout the literature review that having higher scores of self-efficacy [39], self-regulation [42], and sense of coherence had a positive impact on HRQoL. As psychosocial factors are considered to be modifiable, there may be substantial gains to be yielded from paying greater attention to these factors, with a view to improving HRQoL among patients with BC.

This systematic review of 33 studies totaling 5735 participants is the first systematic review to investigate the factors associated with HRQOL in women with BC in the Middle East. Our review has, however, several limitations which need to be considered with caution when interpreting the results. First, despite the rigorous and extensive search strategy with no restrictions on year of publication, there may have been some potentially relevant studies that were eligible, but excluded, because we limited our review to studies published in English. As in any systematic review, publication bias may have affected our findings. Second, the lack of data for some countries may decrease the generalizability of findings to all Middle East regions. Third, due to the heterogeneity among the studies included in the review, only a narrative review was possible. Fourth, the majority of studies had a cross-sectional design, and small sample sizes, implying a low level of evidence, and as such cannot be used to determine causal mechanisms. Finally, although based on the best available data, our review was limited by the quality of studies reported from the Middle Eastern countries. 
Despite these limitations, the current review addressed critical factors that were significantly associated with HRQoL in patients with BC. Emphasis should be given to empowering women through education, as this is a key tool for avoiding unemployment and tackling the psychological impact of BC. Financial aids may also significantly improve the HRQoL of BC patients. Thus, healthcare systems in the Middle East are encouraged to expand interdisciplinary palliative and supportive care services that have the necessary expertise to help financially strained patients to navigate the $\mathrm{BC}$ care pathway. Moreover, there is also a compelling need to provide social support over the long-term to patients with $\mathrm{BC}$. We recommend that clinicians pay attention to modifiable risk factors that have an influence on HRQoL, such as psychological factors. They should also encourage their patients to strengthen their social relationships with family members and friends, to adhere to a healthy diet, and to practice any kind of sport.

\section{Conclusions}

In summary, the present study identified several modifiable and non-modifiable factors that affect HRQoL in women with BC in the Middle East. This study has many implications for practice and provides a framework for establishing policy interventions to more efficiently improve the QoL of women with BC. Healthcare systems in the Middle East are encouraged to develop targeted interventional programs on modifiable factors, particularly socio-demographic, behavioral, and psychosocial ones. Further research on these factors is warranted, preferably through prospective longitudinal studies.

Author Contributions: Conceptualization, R.E.H., L.A.A., V.N. and A.A.; methodology, R.E.H. and L.A.A.; writing —original draft preparation, R.E.H. and L.A.A.; writing—review and editing, R.E.H.; visualization, V.N. and A.A.; supervision, V.N. and A.A.; All authors have read and agreed to the published version of the manuscript.

Funding: This research received no external funding.

Acknowledgments: The authors thank Fiona Ecarnot (EA3920, University Hospital Besancon, University of Franche-Comte, Besancon, France) for editorial assistance.

Conflicts of Interest: The authors declare no conflict of interest.

\section{References}

1. Bray, F.; Ferlay, J.; Soerjomataram, I.; Siegel, R.L.; Torre, L.A.; Jemal, A. Global cancer statistics 2018: Globocan estimates of incidence and mortality worldwide for 36 cancers in 185 countries. CA Cancer J. Clin. 2018, 68, 394-424. [CrossRef] [PubMed]

2. Hirko, K.A.; Soliman, A.S.; Hablas, A.; Seifeldin, I.A.; Ramadan, M.; Banerjee, M.; Harford, J.B.; Chamberlain, R.M.; Merajver, S.D. Trends in Breast Cancer Incidence Rates by Age and Stage at Diagnosis in Gharbiah, Egypt, over 10 Years (1999-2008). J. Cancer Epidemiol. 2013, 2013, 916394. [CrossRef] [PubMed]

3. Elobaid, Y.; Aw, T.-C.; Lim, J.N.W.; Hamid, S.; Grivna, M. Breast cancer presentation delays among Arab and national women in the UAE: A qualitative study. SSM Popul. Health 2016, 2, 155-163. [CrossRef] [PubMed]

4. Osoba, D. Lessons learned from measuring health-related quality of life in oncology. J. Clin. Oncol. 1994, 12, 608-616. [CrossRef] [PubMed]

5. Keegan, T.H.M.; DeRouen, M.C.; Press, D.J.; Kurian, A.W.; Clarke, C.A. Occurrence of breast cancer subtypes in adolescent and young adult women. Breast Cancer Res. 2012, 14, R55. [CrossRef] [PubMed]

6. Collins, L.C.; Marotti, J.D.; Gelber, S.; Cole, K.; Ruddy, K.; Kereakoglow, S.; Brachtel, E.F.; Schapira, L.; Come, S.E.; Winer, E.P.; et al. Pathologic features and molecular phenotype by patient age in a large cohort of young women with breast cancer. Breast Cancer Res. Treat. 2012, 131, 1061-1066. [CrossRef] [PubMed]

7. Hirai, K.; Motooka, H.; Ito, N.; Wada, N.; Yoshizaki, A.; Shiozaki, M.; Momino, K.; Okuyama, T.; Akechi, T. Problem-solving therapy for psychological distress in Japanese early-stage breast cancer patients. Jpn. J. Clin. Oncol. 2012, 42, 1168-1174. [CrossRef] [PubMed]

8. Paraskevi, T. Quality of life outcomes in patients with breast cancer. Oncol. Rev. 2012, 6, e2. [CrossRef] 
9. Schou, I.; Ekeberg, Ø.; Sandvik, L.; Hjermstad, M.J.; Ruland, C.M. Multiple predictors of health-related quality of life in early stage breast cancer. Data from a year follow-up study compared with the general population. Qual. Life Res. 2005, 14, 1813-1823. [CrossRef] [PubMed]

10. Ekwueme, D.U.; Trogdon, J.G. The Economics of Breast Cancer in Younger Women in the U.S. Am. J. Prev. Med. 2016, 50, 249-254. [CrossRef] [PubMed]

11. Keim-Malpass, J.; Levine, B.; Danhauer, S.C.; Avis, N.E. Work-related perceptions and quality of life among breast cancer survivors. Psychooncology 2016, 25, 873-876. [CrossRef] [PubMed]

12. Kwan, M.L.; Ergas, I.J.; Somkin, C.P.; Quesenberry, C.P.; Neugut, A.I.; Hershman, D.L.; Mandelblatt, J.; Pelayo, M.P.; Timperi, A.W.; Miles, S.Q.; et al. Quality of life among women recently diagnosed with invasive breast cancer: The pathways study. Breast Cancer Res. Treat. 2010, 123, 507-524. [CrossRef] [PubMed]

13. Kreitler, S.; Peleg, D.; Ehrenfeld, M. Stress, self-efficacy and quality of life in cancer patients. Psychooncology 2007, 16, 329-341. [CrossRef] [PubMed]

14. Hutter, N.; Vogel, B.; Alexander, T.; Baumeister, H.; Helmes, A.; Bengel, J. Are depression and anxiety determinants or indicators of quality of life in breast cancer patients? Psychol. Health Med. 2013, 18, 412-419. [CrossRef] [PubMed]

15. Leung, J.; Pachana, N.A.; McLaughlin, D. Social support and health-related quality of life in women with breast cancer: A longitudinal study. Psychooncology 2014, 23, 1014-1020. [CrossRef] [PubMed]

16. Papadopoulou, C.; Kotronoulas, G.; Schneider, A.; Miller, M.I.; McBride, J.; Polly, Z.; Bettles, S.; Whitehouse, A.; McCann, L.; Kearney, N.; et al. Patient-reported self-efficacy, anxiety, and health-related quality of life during chemotherapy: Results from a longitudinal study. Oncol. Nurs. Forum. 2017, 44, 127-136. [CrossRef]

17. Herschbach, P.; Berg, P.; Waadt, S.; Duran, G.; Engst-Hastreiter, U.; Henrich, G.; Book, K.; Dinkel, A. Group psychotherapy of dysfunctional fear of progression in patients with chronic arthritis or cancer. Psychother. Psychosom. 2010, 79, 31-38. [CrossRef]

18. Hashemi, S.-M.; Balouchi, A.; Al-Mawali, A.; Rafiemanesh, H.; Rezaie-Keikhaie, K.; Bouya, S.; Dehghan, B.; Farahani, M.A. Health-related quality of life of breast cancer patients in the Eastern Mediterranean region: A systematic review and meta-analysis. Breast Cancer Res. Treat. 2019, 174, 585-596. [CrossRef]

19. Hutton, B.; Salanti, G.; Caldwell, D.M.; Chaimani, A.; Schmid, C.H.; Cameron, C.; Ioannidis, J.P.A.; Straus, S.; Thorlund, K.; Jansen, J.P.; et al. The prisma extension statement for reporting of systematic reviews incorporating network meta-analyses of health care interventions: Checklist and explanations. Ann. Intern. Med. 2015, 162, 777-784. [CrossRef]

20. Stang, A. Critical evaluation of the Newcastle-Ottawa scale for the assessment of the quality of nonrandomized studies in meta-analyses. Eur. J. Epidemiol. 2010, 25, 603-605. [CrossRef]

21. Ahmed, A.E.; Alharbi, A.G.; Alsadhan, M.A.; Almuzaini, A.S.; Almuzaini, H.S.; Ali, Y.Z.; Jazieh, A.-R. The predictors of poor quality of life in a sample of Saudi women with breast cancer. Breast Cancer 2017, 9, 51-58. [CrossRef] [PubMed]

22. Ahrafizadeh, H.; Fayazi, S.; Kord, Z.; Farokhpayam, M.; Farokhpayam, H.; Kiyani, B. The survey quality of life and its related factors among patients with breast cancer who referred to golestan \& amp, Shefa hospitals in ahvaz 2014. Indo Am. J. Pharm. Sci. 2017, 4, 1072-1078.

23. Akel, R.; El Darsa, H.; Anouti, B.; Mukherji, D.; Temraz, S.; Raslan, R.; Tfayli, A.; Assi, H. Anxiety, depression and quality of life in breast cancer patients in the levant. Asian Pac. J. Cancer Prev. 2017, 18, 2809-2816. [PubMed]

24. Akin, S.; Can, G.; Durna, Z.; Aydiner, A. The quality of life and self-efficacy of Turkish breast cancer patients undergoing chemotherapy. Eur. J. Oncol. Nurs. 2008, 12, 449-456. [CrossRef] [PubMed]

25. Alawadi, S.A.; Ohaeri, J.U. Health—related quality of life of Kuwaiti women with breast cancer: A comparative study using the eortc quality of life questionnaire. BMC Cancer 2009, 9, 222. [CrossRef] [PubMed]

26. Albabtain, H.; Alwhaibi, M.; Alburaikan, K.; Asiri, Y. Quality of life and complementary and alternative medicine use among women with breast cancer. Saudi Pharm. J. 2018, 26, 416-421. [CrossRef] [PubMed]

27. Almutairi, K.M.; Mansour, E.A.; Vinluan, J.M. A cross-sectional assessment of quality of life of breast cancer patients in Saudi Arabia. Public Health 2016, 136, 117-125. [CrossRef]

28. Al-Naggar, R.A.; Nagi, N.M.S.; Ali, M.M.S.; Almuasli, M. Quality of life among breast cancer patients in Yemen. Asian Pac. J. Cancer Prev. 2011, 12, 2335-2341.

29. Al-Natour, A.; Al Momani, S.M.; Qandil, A.M.A. The relationship between spirituality and quality of life of jordanian women diagnosed with breast cancer. J. Relig. Health 2017, 56, 2096-2108. [CrossRef] 
30. Bagheri, M.; Mazaheri, M. Body image and quality of life in female patients with breast cancer and healthy women. J. Midwifery Reprod. Health 2015, 3, 285-292.

31. Bayram, Z.; Durna, Z.; Akin, S. Quality of life during chemotherapy and satisfaction with nursing care in Turkish breast cancer patients. Eur. J. Cancer Care 2014, 23, 675-684. [CrossRef] [PubMed]

32. Filazoglu, G.; Griva, K. Coping and social support and health related quality of life in women with breast cancer in Turkey. Psychol. Health Med. 2008, 13, 559-573. [CrossRef] [PubMed]

33. Abu-Saad Huijer, H.; Abboud, S. Health-related quality of life among breast cancer patients in Lebanon. Eur. J. Oncol. Nurs. 2012, 16, 491-497. [CrossRef] [PubMed]

34. Jafari, N.; Farajzadegan, Z.; Zamani, A.; Bahrami, F.; Emami, H.; Loghmani, A. Spiritual well-being and quality of life in Iranian women with breast cancer undergoing radiation therapy. Support Care Cancer 2013, 21, 1219-1225. [CrossRef] [PubMed]

35. Jassim, G.A.; Whitford, D.L. Quality of life of Bahraini women with breast cancer: A cross sectional study. BMC Cancer 2013, 13, 212. [CrossRef] [PubMed]

36. Khalili, N.; Farajzadegan, Z.; Mokarian, F.; Bahrami, F. Coping strategies, quality of life and pain in women with breast cancer. Iran. J. Nurs. Midwifery Res. 2013, 18, 105-111.

37. Ahmad Kiadaliri, A.; Bastani, P.; Ibrahimipour, H. Health-related quality of life of breast cancer patients in Iran: Pooled analysis using generalized estimating equations. Asian Pac. J. Cancer Prev. 2012, 13, 941-944. [CrossRef]

38. Mohammadi, S.; Sulaiman, S.; Koon, P.B.; Amani, R.; Hosseini, S.M. Association of nutritional status with quality of life in breast cancer survivors. Asian Pac. J. Cancer Prev. 2013, 14, 7749-7755. [CrossRef]

39. Moradi, R.; Roudi, M.A.; Kiani, M.M.; Mousavi Rigi, S.A.; Mohammadi, M.; Keshvari, M.; Hosseini, M. Investigating the relationship between self-efficacy and quality of life in breast cancer Patients receiving chemical therapy. Bali Med. J. 2017, 6, 6. [CrossRef]

40. Musarezaie, A.; Zargham-Boroujeni, A. Quality of life and related factors among the women undergoing mastectomy. Iran. J. Nurs. Midwifery Res. 2015, 20, 287-291.

41. Najafi, F.; Nedjat, S.; Zendehdel, K.; Mirzania, M.; Montazeri, A. Self-reported versus proxy reported quality of life for breast cancer patients in the islamic Republic of Iran. East. Mediterr. Health J. 2017, 22, 786-793. [CrossRef] [PubMed]

42. Nikmanesh, Z.; Shirazi, M.; Farazinezhad, F. Examining the predictive role of emotional self-regulation in quality of life and perception of suffering among patients with breast cancer. Middle East J. Cancer 2017, 8, 93-101.

43. Pehlivan, S.; Kuzhan, A.; Yildirim, Y.; Fadiloglu, C. Comfort and quality of life in patients with breast cancer undergoing radiation therapy. J. BUON 2016, 21, 549-555. [PubMed]

44. Rohani, C.; Abedi, H.-A.; Omranipour, R.; Langius-Eklöf, A. Health-related quality of life and the predictive role of sense of coherence, spirituality and religious coping in a sample of Iranian women with breast cancer: A prospective study with comparative design. Health Qual. Life Outcomes 2015, 13, 40. [CrossRef] [PubMed]

45. Safa, A.; Rahemi, Z.; Aghajani, M. Quality of life in patients with breast cancer in Kashan: A cross-sectional study. Life Sci. J. 2014, 11, 141-145.

46. Shakeri, J.; Golshani, S.; Jalilian, E.; Farnia, V.; Nooripour, R.; Alikhani, M.; Yaghoobi, K. Studying the amount of depression and its role in predicting the quality of life of women with breast cancer. Asian Pac. J. Cancer Prev. 2016, 17, 643-646. [CrossRef]

47. Saatci, E.; Akin, S.; Akpinar, E. Do the unmet needs affect the quality of life in breast cancer patients? West Indian Med. J. 2007, 56, 253-257. [CrossRef]

48. Safaee, A.; Moghimi-Dehkordi, B.; Zeighami, B.; Tabatabaee, H.; Pourhoseingholi, M. Predictors of quality of life in breast cancer patients under chemotherapy. Indian J. Cancer 2008, 45, 107-111. [CrossRef]

49. Homaee Shandiz, F.; Karimi, F.Z.; Khosravi Anbaran, Z.; Abdollahi, M.; Rahimi, N.; Ghasemi, M. Investigating the quality of life and the related factors in iranian women with breast cancer. Asian Pac. J. Cancer Prev. 2017, 18, 2089-2092.

50. Sinaei, F.; Zendehdel, K.; Adili, M.; Ardestani, A.; Montazeri, A.; Mohagheghi, M.A. Association Between Breast Reconstruction Surgery and Quality of Life in Iranian Breast Cancer Patients. Acta Med. Iran. 2017, 55, 35-41.

51. Uzun, O.; Aslan, F.E.; Selimen, D.; Koç, M. Quality of life in women with breast cancer in Turkey. J. Nurs. Scholarsh. 2004, 36, 207-213. [CrossRef] [PubMed] 
52. Zamanian, H.; Eftekhar-Ardebili, H.; Eftekhar-Ardebili, M.; Shojaeizadeh, D.; Nedjat, S.; Taheri-Kharameh, Z.; Daryaafzoon, M. Religious coping and quality of life in women with breast cancer. Asian Pac. J. Cancer Prev. 2015, 16, 7721-7725. [CrossRef] [PubMed]

53. Zargani, A.; Nasiri, M.; Hekmat, K.; Abbaspour, Z.; Vahabi, S. A survey on the relationship between religiosity and quality of life in patients with breast cancer: A study in iranian muslims. Asia Pac. J. Oncol. Nurs. 2018, 5, 217-222. [PubMed]

54. Yan, B.; Yang, L.-M.; Hao, L.-P.; Yang, C.; Quan, L.; Wang, L.-H.; Wu, Z.; Li, X.-P.; Gao, Y.-T.; Sun, Q.; et al. Determinants of Quality of Life for Breast Cancer Patients in Shanghai, China. PLoS ONE 2016, 11, e0153714. [CrossRef] [PubMed]

55. Broeckel, J.A.; Jacobsen, P.B.; Balducci, L.; Horton, J.; Lyman, G.H. Quality of life after adjuvant chemotherapy for breast cancer. Breast Cancer Res. Treat. 2000, 62, 141-150. [CrossRef] [PubMed]

56. Broeckel, J.A.; Jacobsen, P.B.; Horton, J.; Balducci, L.; Lyman, G.H. Characteristics and correlates of fatigue after adjuvant chemotherapy for breast cancer. J. Clin. Oncol. 1998, 16, 1689-1696. [CrossRef]

57. Wildes, K.A.; Miller, A.R.; de Majors, S.S.M.; Ramirez, A.G. The religiosity/spirituality of Latina breast cancer survivors and influence on health-related quality of life. Psychooncology 2009, 18, 831-840. [CrossRef]

58. Poleshuck, E.L.; Katz, J.; Andrus, C.H.; Hogan, L.A.; Jung, B.F.; Kulick, D.I.; Dworkin, R.H. Risk factors for chronic pain following breast cancer surgery: A prospective study. J. Pain 2006, 7, 626-634. [CrossRef]

59. Shelby, R.A.; Taylor, K.L.; Kerner, J.F.; Coleman, E.; Blum, D. The role of community-based and philanthropic organizations in meeting cancer patient and caregiver needs. CA Cancer J. Clin. 2002, 52, 229-246. [CrossRef]

(C) 2020 by the authors. Licensee MDPI, Basel, Switzerland. This article is an open access article distributed under the terms and conditions of the Creative Commons Attribution (CC BY) license (http://creativecommons.org/licenses/by/4.0/). 